\title{
Understanding of Grounded-Wire TEM Sounding with Near-Source Configuration
}

\author{
Xue Guo-Qiang*, Chen Wei-Ying, Zhou Nan-Nan, Li Hai and Zhong Hua-Sen \\ Key Laboratory of Mineral Resources, Institute of Geology and Geophysics, Chinese Academy of Sciences, Beijing, China
}

\begin{abstract}
In order to understand the near-source TEM sounding method, the topic on the time domain electromagnetic exploration of deep targets is addressed using a grounded dipole source. At first, the development of the near source TEM is reviewed in this paper, then a new kind of technique capable of detecting geological targets located at deeper levels in the subsurface is proposed using the short-offset transient electromagnetic method (hereafter referred to as SOTEM). The detection abilities of this system, such as the feasibility of near-source detection for SOTEM, the investigation depth of SOTEM and the sensitivity of SOTEM, have been analyzed. The conclusion has been achieved that the proposed method is more applicable and convenient for exploration and could be used to obtain a greater detection depth with higher detection accuracy.
\end{abstract}

Keywords: NIR spectra; Rice; Lead; Copper

\section{Introduction}

TEM is more sensitive to the low resistive anomaly, which poses a pulse waveform with rich frequency-spectral component. Once emitted, it is able to cover all required frequency band with high working efficiency. Based on the classic TEM theory achievement described by famous scholars, such as Wait, Kaufman and Keller, Nabighian and Macnae, Spies, Poddar [1-5], central loop fixed loop and in-loop (with short offset) TEM has been popularly used during last two decades. It is a favorable choice in the geological survey, near-surface engineering exploration, hydrogeological exploration of coal mine and metal mine exploration [6-9]. Because the receiving mode of a loop center transmission is limited by transmission magnetic moment, it is usually able to detect targets buried at only tens to hundreds of meters in depth.

In contrast, the grounded electric source mode, i.e., long offset TEM (LOTEM) can potentially probe the depths of hundreds to thousands of meters. Since 1970, LOTEM has been popularly used in America for deep structure exploration, geothermal investigation and ore prospecting [10-14]. However, the very wide separation between the transmitter and receiver in this configuration may lead to a greater influence on 2-D/3-D structures. Moreover, a larger sending and receiving separation leads to poor signal strength and signal to noise ratio, low the detection accuracy.

Regarding grouned-wire source transient electromagnetic method, radiated filed and primary field can be seperated. If suitble excited-wave form is adopted, the near-source zone deep soundingcan be realized, where field exploration cannot be carried out for the frequency-domain sounding. Although the study and application of short offset transient electro-magnetic has been addressed in Russia $[15,16]$, less attention has been given to the use of Short Offset Transient Electromagnetic in the previous studies for a long time. The high-powered transmitter and short-offset mode system are new developing trends, so more and more researchers are trying to research the near-area mode. A short-offset TEM method was proposed to detect and image resistive targets by Alumbaugh [17]. The short source-receiver offset was employed, so that the measuring points can be more concentrated near the transmitting source. In this method, electric field responses and magnetic field responses are also used. In fact, the measure of electric field in time domain has a potential advantage to detect deeper resistive targets. When the vertical transient current interacts with horizontal layers, the electric field measure is more sensitive to change buildup along the boundaries of electric resistivity. It is believed by Evan [18] and Cuevas [19] that the near source detection is feasible. At present, the time-laps multi-transient electromagnetic method (MTEM) has been successfully applied in the geological exploration with wide attention. Some successful applications in geological exploration are also reported [20-22].

In order to improve the signal to noise ratio of measured data, the integrated technology of TEM survey including a high power transmitter and the short-offset receiving mode (hereafter referred to as SOTEM) is used, i.e., the short distance between transmitter location and receiver position. It has become a kind of new developing orientation. Although this is not a new finding, it is more important to revisit the method for the coming deep ore detecting trend. It is still essential to conduct studies on the near-source response using electric source mode.

\section{Comparisons of Three Kinds of Grounded Source TEM System}

The geological surveys using TEM have different modes, such as LOTEM, MTEM and SOTEM. In order to compare, they are introduced as follows, respectively.

\section{Long off-set grounded source mode TEM}

One of the main features of LOTEM is to have a long distance between transmitter and receiver, and its observation mode is similar to the controlled source audio frequency magnetotelluric method (CSAMT). The typical layout in the field work of LOTEM is shown in Figure 1a. The segment $A B$ denotes the electric dipole source with the length usually from $1 \mathrm{~km}$ to $3 \mathrm{~km}$. $R$ denotes the offset distance between

*Corresponding author: Xue Guo-Qiang, Key Laboratory of Mineral Resources, Institute of Geology and Geophysics, Chinese Academy of Sciences, Beijing, China, E-mail: ppxueguoqiang@163.com

Received July 11, 2013; Accepted September 17, 2013; Published September 23, 2013

Citation: Guo-Qiang X, Wei-Ying C, Nan-Nan Z, Hai L, Hua-Sen Z (2013) Understanding of Grounded-Wire TEM Sounding with Near-Source Configuration J Geophys Remote Sensing 2: 113. doi:10.4172/2169-0049.1000113

Copyright: (c) 2013 Guo-Qiang X, et al. This is an open-access article distributed under the terms of the Creative Commons Attribution License, which permits unrestricted use, distribution, and reproduction in any medium, provided the original author and source are credited. 

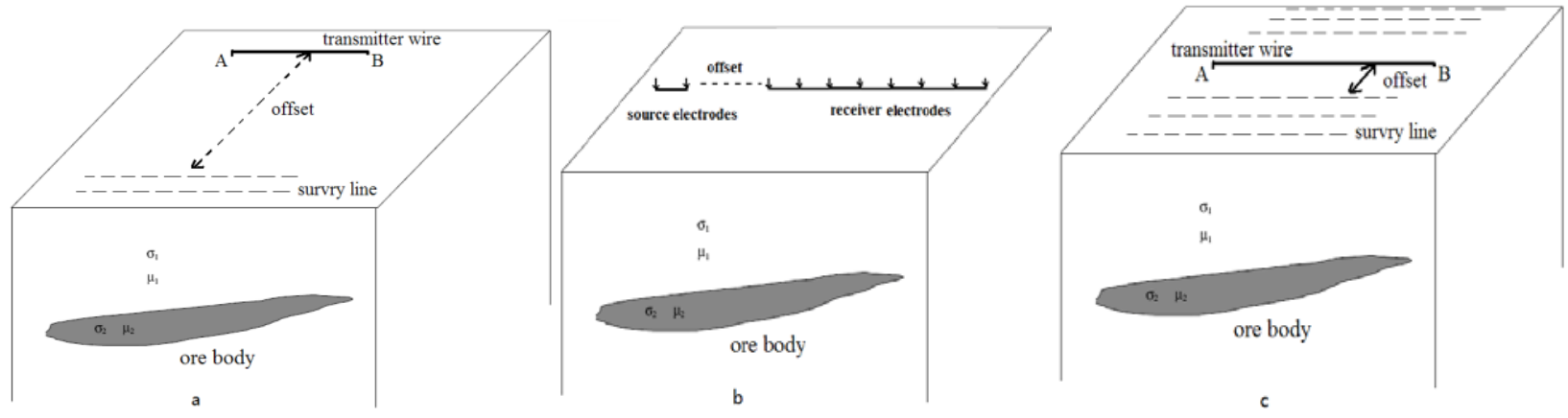

(a) Field work layout of long off-set grounded source mode TEM

(b) The working diagram of the electric source MTEM

(c) Layout of the Grounded Short-offset Electric Source

Figure 1: Three kinds of grounded source TEM system.

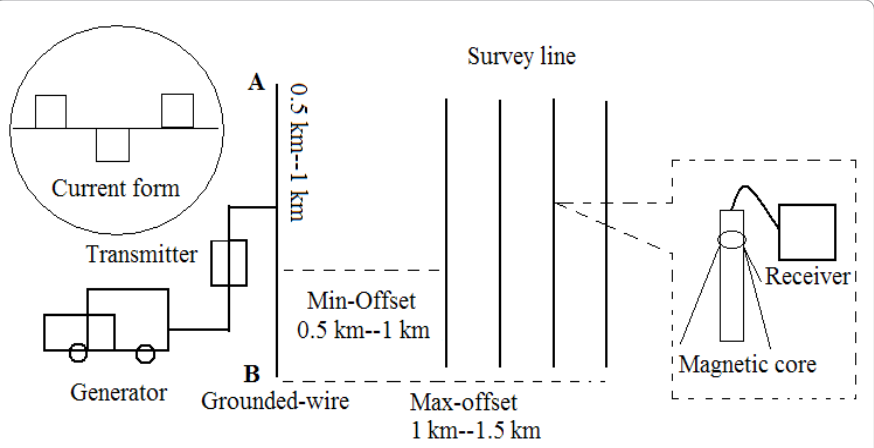

Figure 2: Setup of the short-offset transient electromagnetic method (SOTEM).

the transmitter and receiver. Generally, $R$ is greater than $3-5 \mathrm{~km}$, often up to $20 \mathrm{~km}$. However, because the offset distance is too greater, it may across over multiple different geological structures sometimes, so that the received signal is always poor. Thus, this LOTEM is neither suitable to do the fine detection of geological structures, nor the exploration of complex geological structures.

\section{Multi-channel transient electromagnetic method}

Multi-channel Transient Electromagnetic method (MTEM) was proposed by Wright and Ziolkowski et al. [21]. The MTEM is different from traditional TEM method, because the MTEM work mode is to use the grounded wire source, send random code and do the observation based on multi-channel array, so as to synchronously measure receiving voltage and input sending-current. In addition, its pulse response is obtained through the de-convolution of receiving voltage and input sending-current $[20,21]$. The working diagram of MTEM is shown in Figure $1 \mathrm{~b}$. The main feature of MTEM is shown as the emission source and receiver located on the same measured 006 Cine. Its observation mode is similar to seismic exploration and the received pulse response data are processed as seismic data, which is a great development of data processing in TEM. In this method, the results are displayed using common offset section. The obtained high resistance can indicate the correct horizontal position of gas storage layer well.

\section{Short offset grounded source TEM}

A near-source exploration method, i.e., SOTEM, is proposed in this study, and its layout is shown in Figure 1c. The working procedure includes three contents. (a) After the transmitting wire source (line segment $\mathrm{AB}$ ) is laid, the side-line measurement and exploration are implemented in a certain range of two sides of $\mathrm{AB}$. The requirement of prospecting is the same as that of TEM, and the instruments can be V8, GDP32, PROTEM and so on. The magnetic field signal and electric field signal can be received using a probe and electrodes respectively. (b) Because the received signals are stronger in the near source and intermediate zone, the observation points should be arranged in the above-mentioned areas. On the other hand, the observation points should also be put in a certain offset distance from transmitting field source $\mathrm{AB}$. (c) When the side-line scanning measurement is performed, the single-point measurement is taken with literal movement, or several points can be synchronously measured using multiple channels. Single or many times spatial coverage can all be done using single-point moving arrangement.

\section{Near-Source Exploration System}

In Figure 1c, a kind of SOTEM configuration is proposed by authors, and its setup is shown in Figure 2. The transmitter and receiver are separated with short offset $(1000 \mathrm{~m}-1500 \mathrm{~m})$, the transmitting wire source (line segment $\mathrm{AB}$ with a length of $500 \mathrm{~m}-1000 \mathrm{~m}$ ) is grounded to earth, while a step-wave current is generated by a high-powered generator. The induced voltage data are collected by a magnetic core, whereas the electric field component (Ex) data are collected by an electrode. An observation area is selected around the near-area of electrical source for detecting underground target within the buried depth of $2000 \mathrm{~m}$.

The V8 instrument made in Canada can be adopted in SOTEM system, and the SB-7K magnetic probe made in China is configured to collect induced transients electromagnetic signal. The effectively receiving area is $40000 \mathrm{~m}^{2}$. After the experimental selection, a set of working parameters are determined. The current step can be used to emit to a transmitter with the transmitting current of $20 \mathrm{~A}$. The range of time delay can be selected as $0.087 \mathrm{~ms}-100 \mathrm{~ms}$. The measurement of each receiver point can be finished within 7 minutes.

The proposed SOTEM configuration has many advantages as followings:

(1) High work efficiency: Compared with square loop source, the wire source can be easily laid, especially in mountainous areas, where a transmitting device is accompanied by two or more equipments used for simultaneous data acquisition with SOTEM; in addition, the survey can be carried out simultaneously on both right and left sides of the transmitter. It is very convenient and high efficient in the mountain 
Citation: Guo-Qiang X, Wei-Ying C, Nan-Nan Z, Hai L, Hua-Sen Z (2013) Understanding of Grounded-Wire TEM Sounding with Near-Source Configuration. J Geophys Remote Sensing 2: 113. doi:10.4172/2169-0049.1000113

Page 3 of 5

geological exploration, because the wire can be laid through the ravine sidewalk in mountains. The shorter source-receiver offset in SOTEM method is employed, so that the measure points can be more focused on the anomaly below the source.

(2) High resolution: SOTEM has a smaller volume but higher resolution than LOTEM; furthermore, SOTEM excites not only the horizontal component of an electric field, but also the vertical component of the field on a geo-electric interface, whereas the loop source TEM excites only the horizontal component of electric field. Additionally, SOTEM has a high penetrability in the high-resistivity medium and a high resolution in the low-resistivity medium.

(3) Greater detection depth: The detection depth can be determined mainly by time and earth geo-electric structure. However, this is less related to the source-field point offset distance. In such a configuration, the near-area survey point can receive stronger signals than far area, which can subsequently yield field data characterized by higher signalto-noise ratios and also provide deeper target information.

\section{Feasibility of Near-Source Detection for SOTEM}

In the uniform half-space, the responses of controlled source audio-frequency magnetotelluric method (CSAMT) in the near area are shown by the following mathematical relations [2];

$$
\begin{aligned}
& E_{x}=\frac{I d l}{4 \pi \sigma} \frac{1}{r^{3}}(3 \cos 2 \theta+1) \\
& E_{y}=\frac{3 I d l}{4 \pi \sigma} \frac{1}{r^{3}} \sin 2 \theta \\
& H_{x}=-\frac{I d l}{4 \pi} \frac{1}{r^{2}} \sin 2 \theta \\
& C_{y}=\frac{I d l}{2 \pi r^{2}} \cos 2 \theta \\
& H_{z}=\frac{I d l}{4 \pi} \frac{1}{r^{2}} \sin \theta
\end{aligned}
$$

where $I d l$ is the dipole moment, $I$ is the current, $d l$ is the length of dipole, $r$ is the separating distance, $\theta$ is the angle between transmitter line direction and offset direction, and $\sigma$ is the earth conductivity.

If the excitation wave form is similar to those in Figure 3a (Normal
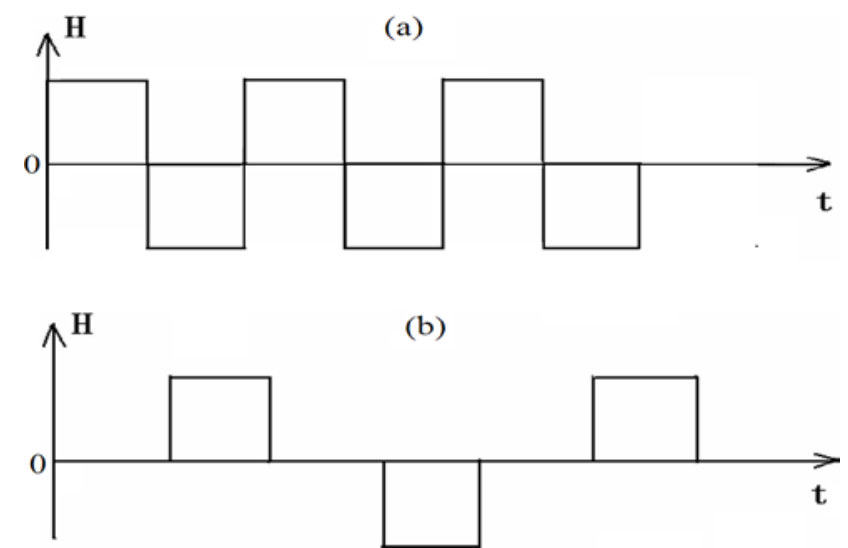

Figure 3: A comparison between (a) normal CSAMT bipolar square wave form and (b) normal TEM step excitation wave form.

\begin{tabular}{|c|c|c|c|c|}
\hline $\mathrm{I}(\mathrm{A}) \rho(\Omega \cdot m)$ & $L(m)$ & $r(m)$ & $d_{f}(m)$ & $d_{n}(m)$ \\
\hline 1.0 & 20.0 & 100.0 & 212.7 & 243.1 \\
\hline 5.0 & 50.0 & 300.0 & 526.4 & 625.2 \\
\hline 10.0 & 100.0 & 500.0 & 845.9 & 1012.0 \\
\hline 20.0 & 300.0 & 1000.0 & 1574.5 & 1910.9 \\
\hline 50.0 & 1000.0 & 4000.0 & 3783.3 & 5084.2 \\
\hline
\end{tabular}

Table 1: Detectable depth values of far-area mode and near-area mode.

CSAMT bipolar square wave form), the primary and secondary fields cannot be separated, and there is no relationship between magnetic field component and earth conductivity in Equation 3-5, indicating that CSAMT cannot work well in the near-source area. On the other hand, if the excitation wave form is a step-wave type as shown in Figure 3b, the secondary field response is surveyed after turning off the primary field, which means that TEM near-source area sounding can be produced if a step-wave form is adopted.

\section{The Investigation Depth of SOTEM}

The downward diffusion depth $(d)$ of TEM refers to the maximum depth travelled by TEM eddy current field at a given time, which can be expressed as follows [4];

$$
d=\frac{4}{\sqrt{\pi}} \sqrt{t / \rho \mu_{0}}
$$

where $t$ is the time and $\rho$ is the resistivity of investigated geological material. Apparently, the TEM diffusion depth is mainly related to the time and geological-electrical structure, rather than the transmitterreceiver separating distance.

In practice, considering the performance of equipment, receiver sensitivity, accuracy of measurement, noise intensity and geologicalelectrical parameters, the detection depth $\left(d_{f}\right)$ of far-area mode (i.e., LOTEM) is often estimated using the following equation [4];

$$
d_{f}=0.28\left(\frac{I \rho L}{\eta}\right)^{1 / 4}
$$

Similarly, the detection depth $\left(d_{n}\right)$ of the near-area mode (e.g., SOTEM) is estimated using the mathematical relation below:

$$
d_{n}=0.48\left(\frac{\operatorname{Ir} \rho L}{\eta}\right)^{1 / 5}
$$

In Equation (4) and (5), $\eta$ is the voltage resolution precision of measuring instrument, $\rho$ is the resistivity of detected geological structure, $L$ is the length of transmitting source line $\mathrm{AB}$, and $r$ is the transmitter-receiver separation. The depth values calculated using Equation (7) and (8) are listed in Table 1. Where, $d_{f}(m)$ denotes the detection depth using a near-area mode, and $d_{n}(m)$ denotes that using a far-area mode. It can be seen from the table that the detection depth using a near-area mode is generally deeper compared with the depth obtained using the far-area mode.

\section{Sensitivity of SOTEM}

It is well known that the resistance structure is difficult to be delineated by the conventional loop TEM methods [23]. The main reason is that the response of TEM methods relies on the inductive effect of horizontal source fields. Even though the LOTEM method can generate both horizontal and vertical transient magnetic fields using grounded electric dipole source, its magnetic field responses are also not strongly induced by underground thin resistors structure [13]. This is because the response magnetic fields are mainly from the horizontal 
current flow. Obviously, the horizontal current flow cannot penetrate the ground objects through inductive coupling well.

In order to compare the sensitivity of SOTEM and LOTEM to the geological structure with the thin thickness and high resistance, a survey example of the underground geological structure, of which the thickness of geological model is $5 \mathrm{~m}$ and the apparent resistivity is 300 $\Omega . m$, is numerically carried out. The apparent resistivity of the rock around the thin geological structure is $100 \Omega . m$. The length of source wire in LOTEM is $200 \mathrm{~m}$ and the transmitting line length in SOTEM is also $200 \mathrm{~m}$. In the both, the transmitting electric currents are 10A, the frequency is $30 \mathrm{~Hz}$, and the range of time sampling is $0.1087-7.1015 \mathrm{~ms}$.

The apparent resistivity sensitivity of geological model with and without the geological layer of the thin thickness and high electric resistor are calculated using SOTEM and LOTEM respectively, and the results are shown in Figure 4.

Solid line is the error between apparent resistivity of geological model with and without thin thickness, high resistor for SOTEM method. Dash line is the error between apparent resistivity of geological model with and without thin thickness, and high resistor for LOTEM method. It is found in Figure 4 that the sensitivity of SOTEM is greater than that of LOTEM at late time. It is indicated that the resolution of SOTEM is better than that of LOTEM. This kind of the phenomenon can be explained as follows. The electric current source in SOTEM can lead to the vertical and horizontal currents in geological structures, and the interaction between the vertical current and horizontal geological anomaly will produce the cumulative charge along the boundary of the object with high resistivity. Because the response to the electric current source is sensitive to the cumulative charge, the mode of SOTEM has a better resolution to the thin and resistance geological layer.

In fact, the application of LOTEM can only detect the conductive layer and is not sensitive to resistance structure. In contrast, SOTEM can not only detect the thin high resistivity geological structure, but also detect the thin low resistivity geological structure.

\section{Discussion and Conclusions}

Because the receiving mode of a loop center transmission is limited by the transmission magnetic moment of loop, the several tens to several hundred meters of earth depth can only be detected generally. But the application of TEM with grounded electric source can detect rather greater depth than the former, so, TEM with the grounded-wire source has become a hot developing technology in geological exploration. However, the LOTEM technique its magnetic field responses cannot be strongly excited by underground thin resistors because the magnetic field only depends on the horizontal electric current penetrating into

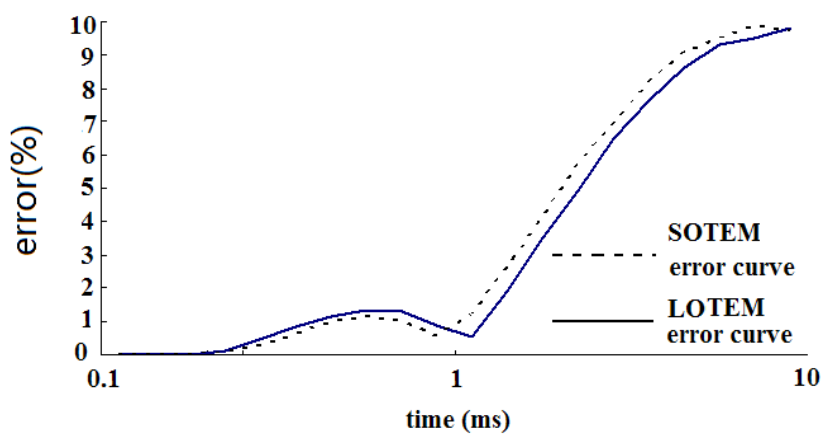

Figure 4: Sensitivities of SOTEM and LOEM to the thin and high- resistivity geological layer. the ground through inductive coupling, in addition, a large distance between transmitter $(\mathrm{Tx})$ and receiver $(\mathrm{Rx})$ may lead to the reliability decrease on geological interpretation of 2-D or 3-D structures, Therefore, it is an important basic issue to study the detection ability of TEM with the short offset.

Theoretically, if step-wave form is adopted as the excitation wave, the secondary field response is surveyed after turning off the primary field, then, TEM near-source area sounding that can be produced. Thus the weak signal disadvantage of LOTEM can be well overcome. SOTEM has few additional effects, bigger exploration earth's depth and stronger exploration ability to geological section and limited conductor. This not only realizes multiple time cover measures of the detected ground geological unit, but also the 3-D exploration of TEM.

Totally, this study presents a kind of the strategy detecting the geological targets in the deeper stratum using the sophisticated detection methods and treatment technologies of the TEM, and it is also fast, efficient and easy to implement. The main contents are to do the transient electromagnetic detection in the near source as well as make the processing and explanation of the observational data with the whole field area theory. Because the observation point is closed to emission source, the non-dipole effect must be adjusted, i.e., the emission wire is considered as the sum of numerous dipoles, and the field response of the observation point equals the sum of those field response induced by each dipole of emission source wire. The proposed method is helpful to obtain more accurate information of the shape, size and location of an underground target body and possess the important significance to the detailed study of geological structures. This innovative approach can be potentially employed in a broader scientific study such as engineering and geological exploration projects.

\section{Acknowledgments}

This research was supported by the State Major Basic Research Program of the People's Republic of China (2012CB416605) and Natural Science Foundation of China (NSFC) (41174090 41174108, and 41130419). Many thanks to Dr. Patrick who is working in University of Ghana, for improving the English.

\section{References}

1. Wait JR (1951) Transient electromagnetic propagation in a conducting medium Geophysics 16: 213-221.

2. Kaufman AA, Keller GV (1983) Frequency and Transient Soundings, Elsevier, Netherlands.

3. Nabighian M, Macnae J (1991) Time domain electromagnetic prospecting methods in Electromagnetic Methods in Applied Geophysics. Soc of Explor Geophys 2.

4. Spies BR (1989) Depth of investigation in electromagnetic sounding methods. Geophysics 54: 872-888.

5. Poddar M (1983) A rectangular loop source of current on multilayered earth Geophysics 48: 107-109.

6. Zhang Z, Xiao J (2001) Inversion of surface and borehole data from large-loop transient electromagnetic system over a 1-D earth. Geophysics 66: 1090-1096.

7. Singh NP, Mogi T (2005) Electromagnetic response of a large circular loop source on a layered earth: A new computation method. Pure Appl Geophys 162: 181-200.

8. Xue GQ, Yan YJ, Cheng JL (2011) Researches on detection of 3-D underground cave based on TEM technique. Environ Earth Science 64:425-430.

9. Xue GQ, Bai CY, Yan S, Greenhalgh S, Mei-fang Li, et al. (2012) Deep sounding TEM investigation method based on a modified fixed central-loop system. Journal of Applied Geophysics 76: 23-32.

10. Yang Sheng (1986) A single apparent resistivity expression for long-offset transient electromagnetic. Geophysics 51: 1291-1297.

11. Strack KM, Luschen E, Kota AW (1990) Long-offset transient electromagnetic 
Citation: Guo-Qiang X, Wei-Ying C, Nan-Nan Z, Hai L, Hua-Sen Z (2013) Understanding of Grounded-Wire TEM Sounding with Near-Source Configuration. J Geophys Remote Sensing 2: 113. doi:10.4172/2169-0049.1000113

(LOTEM) depth soundings applied to crustal studies in the Black Forest and Swabian Alps, Federal Republic of Germany. Geophysics 55: 834-842.

12. Vladimir AH, Druskin VL, Kinizhnerman LA, Strack LM (1992) Interpretation of 3-D effects in long-offset transient electromagnetic (LOTEM) soundings in the Munsterland area/Germany. Geophysics 57: 1127-1137.

13. Hordt A, Muller M (2000) Understanding LOTEM data from mountainous terrain. Geophysics 65: 1113-1123.

14. Horad A, Scholl C (2004) The effect of local distortions on time-domain electromagnetic measurements. Geophysics 69: 87-96.

15. Goldman MM (1977) Transient electromagnetic field of a disk in a stratified medium Geol. Geofiz.

16. Rabinovich BI (1977) Two layer sounding curves for the transient horizonta magnetic component of the field in the far and near zone, Geol. Geofiz 11:148152

17. Alumbaugh D (2002) Research proposal on 3D numerical analysis of the Grounded Electric Source. Transient Electromagnetic Geophysical Method.
18. Evan SU (2005) On the Physics of Galvanic Source Electromagnetic Geophysical Methods for Terrestrial and Marine Exploration. Ph.D. thesis University of Wisconsin-Madison, USA.

19. Cuevas $\mathrm{NH}$, Alumbaugh $\mathrm{D}$ (2009) Near source response of a resistive layer to vertical electric dipole excitation. October 25-30, SEG Houston, International Exposition and Annual meeting 794-798.

20. Wright D, Ziolkowski A, Hobbs B (2002) Hydrocarbon detection and monitoring with a multi component transient electromagnetic (MTEM) survey. The Leading Edge 21: 852-864

21. Ziolkowski A, Hobbs BA, Wright D (2007) Multi-transient electromagnetic demonstration survey in France. Geophysics 72: 197-209.

22. Ziolkowski (2010) Short-offset transient electromagnetic geophysical survey. United State Patent Application Publication.

23. Müller M, Hördt A, Neubauer FM (2002) Internal structure of Mount Merapi, Indonesia, derived from long-offset transient electromagnetic data. Journal of Geophysical Research solid Earth 107: 2187. 